Scenes from Postgraduate Life

\title{
Big is best?
}

Working in a hospital of 900 beds in the 60 s and 70 s we constantly agonized over the need to be extra careful about human relationships. It was the time of Schumacher and 'Small is Beautiful', the closure of caring cottage hospitals, and a report from Manchester which showed that hospitals were dangerous places to work in and created a high degree of anxiety among staff. At that time we had a hospital secretary who regarded one of the most important parts of his job as building bridges between staff and patients, and on the whole we felt that size was not a bar to decent communication and a friendly environment. The fact that we tended to be apologetic about impersonality perhaps illustrates the importance we attached to attitudes. It will be interesting to see, with the current emphasis on money and efficiency, whether unit managers (just another name for unit administrators?) will consider human relationships as part of their role.

We were planning a new district hospital, and because we had plenty of space could afford a certain amount of grand thinking. In fact we felt that three or four units of not more than 600 beds, each with separate administrators could be accommodated on the site, in which would be grouped, for example, medicine, surgery, paediatrics and maternity; specialties such as accident and emergency, orthopaedics, ear, nose and throat, ophthalmology, neurology and neurosurgery; geriatrics; and psychiatry. Needless to say 25 years later there is no new hospital, only a new casualty and outpatient department. Seeing some of the district hospitals that have been built I'm not sure whether to be pleased or disappointed.

Due to accidents of history many districts have more than one hospital, especially in the Thames Regions, often separated both geographically and functionally. Some are too small or too specialized to support more than minimal services. A tidy solution becomes more and more difficult, but may have to be imposed on grounds of both economy and efficiency. The long-term effects are not always foreseen. It seems logical to have a single accident and emergency department to serve a district not solely on economic grounds but to concentrate the required expertise. But a hospital which loses such a department is immediately robbed of its mix of cases, and medical emergencies lose the spontaneous quality which makes them so valuable for training in the pre-registration year. Loss of approval for doctors in the latter category will alter the nature of the hospital's work, and there is a danger of the hospital reverting to the understaffed geriatric/ chronic sick pattern of the pre-NHS workhouse infirmary.

A somewhat analogous example is the desire to concentrate pathological services (and to a lesser extent radiology) on a single site. This has obvious benefits in terms of technology and expertise, but where laboratories serve more than one hospital there are practical difficulties in transporting specimens and communicating results. In addition, junior staff suffer because of lack of contact with laboratory personnel; telephone conversations are an unsatisfactory way of developing mutual respect and confidence.

The same argument can be extended to concentration of specialists on one site: junior doctors at another hospital may be disadvantaged by their inability to learn from expert advice and discussion on the spot because it is more convenient to send the patient to the expert. Also it may not be possible to provide more than limited facilities for postgraduate medical education (a single room with a small number of reference textbooks) at some of the hospitals in the district, and this causes problems with approval of posts for training. It is impractical to expect junior staff to travel regularly to another hospital for staff rounds and lunchtime or out-of-hours meetings. Quality declines and some posts tend to be filled by repeated locums.

A District which has several hospitals employs consultants who work on different sites and may have beds at more than one hospital. This means there are supporting junior staff at each hospital, admittedly working for more than one consultant. Since salaries are the major component of the District's budget, and given the need to reduce posts at registrar and to a lesser extent at SHO grades, there is an advantage in the single site hospital. The hospital with the widest spread of departments is obviously the one in the running for the latest piece of special equipment. And the efficiency with which services are delivered does not automatically exclude humanity.

There are arguments for and against the view that the bigger the hospital the better the service, but the trend towards large may be dictated in the final instance by the present economic crisis. How much will new managers have to consider what seems to be an inevitable fact of the NHS of the future - that big is best?

This fits with professional aspirations - at least by hospital consultants. Among physicians, various 
groups have been calling for at least one, and sometimes two, appointments per District in their particular specialty, for example, gastroenterology, rheumatology, physicians with an interest in diabetes, cardiovascular physicians. No doubt a case can be made for each, though some smack a little of special pleading. There are dangers, however: junior staff have pointed out that if the number of physicians with a special interest were to be increased, the emergency work would be so diluted that they would get insufficient exposure. Do District hospitals need to be staffed entirely by specialists? In the North East Thames Region we now have only five senior registrar posts out of some 270 in general internal medicine alone, all the rest having a special interest, so it looks as though the trend has to be accepted. But the appointment of specialists generates more work and is expensive. I hear that one reason why there are unfilled consultant posts around the country is because Districts cannot afford the additional cost of up to $£ 250000$ which goes with the appointment.

In future no doubt we will have to justify new posts on cost effective grounds if we want to convince the managers. Physicians with an interest in diabetes, for $\cong$ instance, may have cause to be upset that $12 \%$ of $\vec{z}$ Districts do not at present have a specialist service. But $\stackrel{\varnothing}{\AA}$ before such a deficiency is made good they may have to $c$ mount an investigation to see in what way outcome $\overrightarrow{\vec{B}}$ differs between districts with and without diabetologists. When our coronary care unit was temporarily closed for upgrading we were advised not to admit patients with suspected myocardial infarcts. We $\overparen{\mathbb{}}$ should have seized the opportunity to see whether its $\varrho$ absence made any difference to outcome, as judged by क objective tests rather than by prejudiced opinions $\vec{\circ}$ which now make such an experiment impossible.

The argument as to what type of specialists we should be training tends to be carried on by people with a vested interest. Pressure, frequently from outside the profession, is shifting the emphasis away from acute services, and if we want to preserve them we will have to provide irrefutable statistical evidence $N$ of the need-and that is not easy.

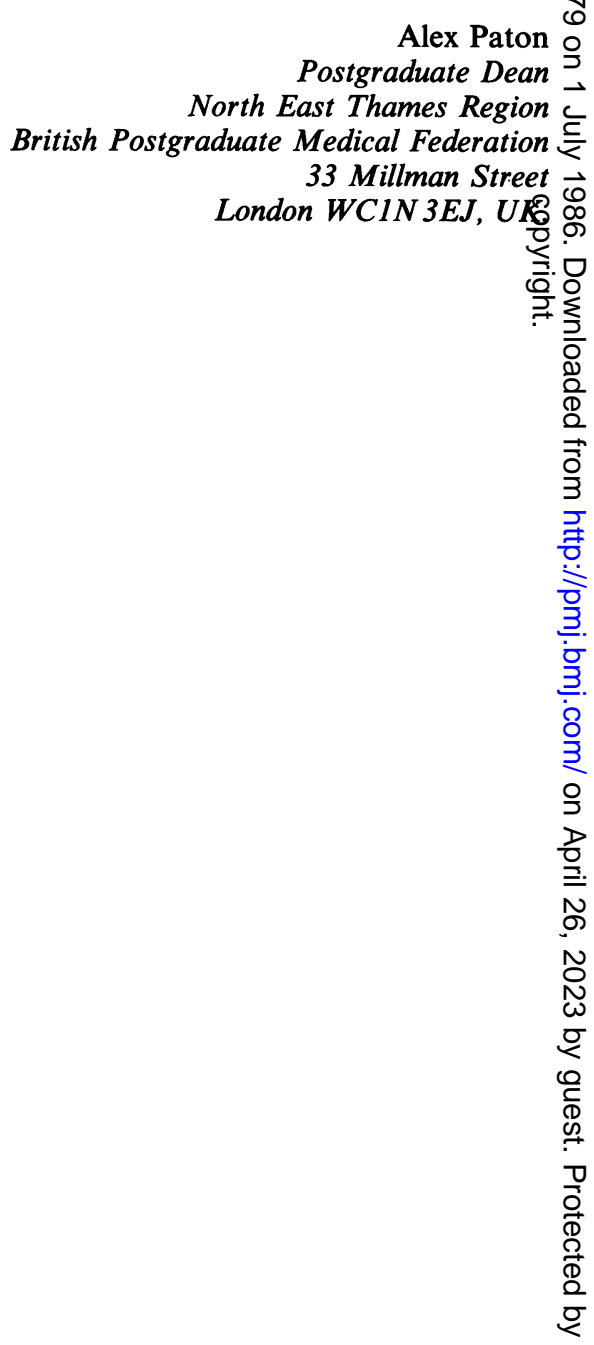

